\title{
When neurological symptoms hides a nephrologic pathology and a cardiac abnormality
}

\author{
C. Muller $^{1}$, B. Faller ${ }^{1}$, F. Sellal ${ }^{2}$, F. Chantrel ${ }^{1}$
}

${ }^{1}$ Nephrology department, Civil Hospital, Colmar, France;
${ }^{2}$ Neurology department, Civil Hospital, Colmar, France.
E-mail: clotilde-muller@hotmail.fr

Received 2 May 2011; revised 7 June 2011, accepted 15 June 2011.

\begin{abstract}
We bring report the case of a young woman who presents with neurological symptoms, and to which the practised investigations led to the discovery in chain of unexpected pathologies. These neurological disorders were revealing of the picture associating a nephrotic syndrome and a patent foramen ovale. This foramen was the major route of a rain of emboles ring peripheral favored by the nephrotic syndrome towards the cerebral hemispheres.
\end{abstract}

Keywords: Edema; Hypercoagulable State; Patent Foramen Ovale; Nephrotic Syndrome; Stroke; Venous Thrombosis

\section{INTRODUCTION}

This case shows a young woman with neurologic symptoms, in whom further investigations lead to find unexpected disorders. The association of an anatomical abnormality and a renal pathology can do many damages.

\section{CASE REPORT}

A thirty-seven-year-old woman was admitted on 24 th December 2007 to the emergency room for acute right hemiparesis and central facial diplegia with anomic aphasia. A general exam showed only high blood pressure (161/84 mmHg), and peripheric edema. She had a recent history of ankles edema in August 2007. She first consulted her general practician in October, who started a treatment for high blood pressure without a specific investigation of edema status (Valsartan (160 mg/d), hydrochlorothiazide $(12.5 \mathrm{mg} / \mathrm{d})$ and Rilmenidine $(1 \mathrm{mg} / \mathrm{d})$ : we don't know why the three treatments have been started at the same time). She didn't take any contraception.

The patient had a past history of hypothyroidism that was stabilized with levothyroxine sodium treatment, asymptomatic biliary lithiasis, and she had smoked 15 cigarettes per day since 1984. She was an office and cloakroom cleaner in a factory that used aluminium.

The biology workup on 12/24/07 showed hypoproteinemia, $48 \mathrm{~g} / \mathrm{l}$; hypoalbuminemia, at $21 \mathrm{~g} / \mathrm{l}$; creatinine, $0.92 \mathrm{mg} / \mathrm{dl}$ (82 $\mu \mathrm{mol} / \mathrm{l})$; Modification of Diet in Renal Disease formula (MDRD), $73 \mathrm{ml} / \mathrm{min} / 1.73 \mathrm{~m}^{2}$; protenuria, $4.96 \mathrm{~g} / \mathrm{d}$; Thyroid-Stimulating Hormone, $2.34 \mathrm{mU} / \mathrm{l}$ C-reactive protein normal; Coagulation, immunology and microbiology were normal.

Cerebral Computed Tomography (CT) was normal. Cerebral Magnetic resonance Imaging (MRI), on T2weighted and diffusion sequences, showed multiple small deep cerebral hyperintensities, mainly located on the left basal ganglia and white matter, and a left cortical insular lesion (Figure 1). These lesions evoked multiple recent ischemic lesions, suggestive of a cardiac embolic mechanism or cerebral vasculitis.

Cervical and transcranial Doppler ultrasonography showed no abnormality.

The electrocardiogram (ECG) and 24 hour ECG showed no arrhythmia. Transesophageal echocardiography showed a patent foramen ovale with contrast and in a Valsalva manoeuvre, without any cardiac thrombus, or aneurysm, nor signs of endocarditis. Aortis seemed normal, and there was neither valvulopathy nor any abnormality on the left atrium. The transthoracic echography showed no hypokinetic portion with a normal cardiac function. Initial treatment consisted of continuous unfractioned heparin at hypocoagulating doses.

As she presents multiple recent ischemic cerebral lesions and a nephritic syndrome, we linked these symptoms by the discovery of a foramen ovale at the transesophageal echocardiography. There was still to find if there was thrombosis somewhere.

Deep venous thrombosis was investigated using venous Doppler of the legs and renal veins and lung perfusion scintigraphy, which were negative but undertaken after initiation of heparin therapy. Renal biopsy showed membranous glomerulopathy.

After a few days, she had recovered normal neu- 


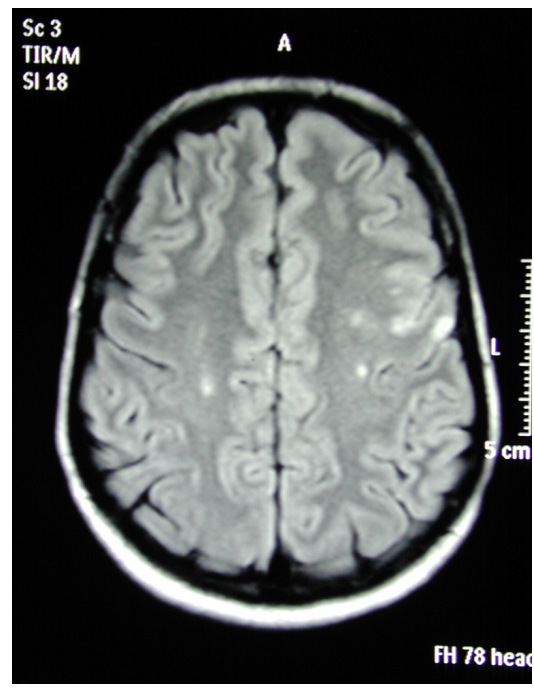

Figure 1. Cerebral MRI: diffusion sequences, showed multiple small deep cerebral hyperintensities, mainly located on the left basal ganglia and white matter, and a left cortical insular lesion.

rologic functions.

\section{DISCUSSION}

Patent foramen ovale (PFO) affects $25 \%$ of the population [1]. Nearly half of patients who are less than 50 years of age with a history of stroke have a persistent PFO [2].

Nephrotic syndrome is a hypercoagulable state and has a frequent complication: venous or arterial infarction in various organs. Arterial thrombosis, mainly of the femoral artery, is rare and ischemic stroke has been more rarely described, sometimes as an initial manifestation [3]. Moreover, co-occurrence of nephrotic syndrome and patent foramen ovale leading to a cerebral infarction is exceptional. Since there is a high risk of deep venous thrombosis in the renal vein and pulmonary artery, anticoagulation by anti-vitamin K (AVK) is usually the beginning treatment in patient with major nephrotic syndrome when albuminemia is under $2.0 \mathrm{~g} / \mathrm{dl}$. In our patient, although no venous thrombosis was found but it is often the case, as the Doppler is not realised in the same time of the stroke happened and many veins haven't been explored (pelvic veins...). The presence of both patent foramen ovale and bilateral and widespread cerebral strokes of the same age strongly suggested that venous thrombosis was the origin of multifocal embolic ischemic stroke.
Initial treatment with heparin followed by AVK was highly effective on the neurologic signs. We continued AVK treatment for 3 months, assuming a venous thrombosis hypothesis, waiting for corticosteroids action as first-line treatment of nephrotic syndrome.

Prima facie PFO in the presence of multiple ischemic strokes requires an anticoagulant therapy. Nevertheless, the PICS study [4], which compared aspirin with AVK, showed no reduction in recurrence or death under AVK vs aspirin. In young patients, the risk of recurrent stroke is low (1\% - 2\% per year). Closing PFO seems necessary only if there is a recurrence with treatment, thromboembolic disease, or if the patient refuses to take medications [5].

\section{CONCLUSIONS}

The presence of both patent foramen ovale and bilateral and widespread cerebral strokes of the same age strongly suggested that venous thrombosis was the origin of multifocal embolic ischemic stroke.

This double discovery of a membranous glomerulopathy and patent foramen ovale from an initial cerebral infarction is exceptional.

\section{REFERENCES}

[1] Wechsler, L.R. (2008) PFO and stroke: What are the data? Cardiology in Review, 16, 53-57 doi:10.1097/CRD.0b013e31815771e4

[2] Mesa, D., Franco, M., Suárez de Lezo, J., Munoz, J., Rus, C., Delgado, M., Ruiz, M., Pan, M., Romo, E., Vallès, F., Vinals, M. and Bescansa, E. (2003) Prevalence of patent foramen ovale in young patients with cerebral ischemic accident of unknown origin. Revista Espanola de Cardiologie, 56, 662-668. doi:10.1157/13049647

[3] Marsh, E.E., Biller, J., Adams Jr, H.P. and Kaplan, J.M. (1991) Cerebral infarction in patients with nephrotic syndrome. Stroke, 22, 90-93. doi:10.1161/01.STR.22.1.90

[4] Homma, S., Sacco, R.L., Di Tullio, M.R., Sciacca, R.R., and Mohr, J.P., for the PFO in cryptogenic stroke study (PICSS) investigators. (2002) Effect of medical treatment in stroke with patient with patent foramen ovale: Patent foramen ovale in cryptogenic stroke study. Circulation, 105, 2625-2631. doi:10.1161/01.CIR.0000017498.88393.44

[5] Albucher, J.F., Chaine, P., Mas, J.L., Moulin, T., Rodier, G., Cohen, A., Derumeaux, G., Lusson, J.R., Malerque, M.C., Rey, C., Société Française de Cardiologie. (2007) Consensus about indications of endovascular closure of the patent foramen ovale after an ischemic brain stroke. Archives des Maladies du Cœur et des Vaisseaux, 100, 771-774. 